\title{
In Vitro Evaluation of Aerosols Delivered via the Nasal Route
}

\author{
Katia K El Taoum MD, Jinxiang Xi PhD, JongWong Kim PhD, and Ariel Berlinski MD
}

BACKGROUND: Infants and young children are obligate nose breathers; therefore, a transnasal route seems the logical delivery method of inhaled aerosols. The efficiency of aerosol delivery depends on several factors, such as interface, type of nebulizer, and patient age and breathing pattern. We hypothesized that the use of a vibrating mesh nebulizer, a tight-fitting face mask, and a head model and breathing pattern of an older child would result in a higher lung dose. We also hypothesized that the use of an anatomically correct model would more accurately reflect lung dose than models that do not include airways. METHODS: A model comprising a breathing simulator and an anatomically correct model of a 7-month-old infant and a 5-y-old child with an interposed collection filter (lung dose) were used. Breathing patterns of a newborn, infant, and child were used with 7 interfaces. A continuous output and a vibrating mesh nebulizer were loaded with albuterol sulfate solution $(5 \mathrm{mg} / 3.5 \mathrm{~mL}$ ) and operated for $5 \mathrm{~min}$. Albuterol mass was determined via spectrophotometer $(276 \mathrm{~nm})$. RESULTS: Lung dose varied between 0 and $3 \%$. The jet nebulizer was more efficient than the vibrating mesh nebulizer. The front-loaded mask was the most efficient interface. We also found that higher tidal volumes were associated with higher lung doses and that the use of a larger airway model resulted in a lower lung dose. Finally, the model showed a good correlation with in vivo data and rendered lung doses severalfold lower than previous data obtained with oral models. CONCLUSIONS: Careful pairing of the aerosol generator and interface is very important during transnasal aerosol delivery. Key words: aerosol delivery; nasal interface; nebulizer; tidal volume; airway model; face mask; lung dose. [Respir Care 2015;60(7):1015-1025. (C 2015 Daedalus Enterprises]

\section{Introduction}

Aerosol medicine is one of the mainstays of treatment for respiratory diseases. Its use is increasing in infants and

Drs Taoum and Berlinski are affiliated with the Department of Pediatrics, Pulmonology Section, University of Arkansas for Medical Sciences, Little Rock, Arkansas. Drs Xi, Kim, and Berlinski are affiliated with the Department of Mechanical and Biomedical Engineering, Central Michigan University, Mount Pleasant, Michigan. Dr Berlinski is also affiliated with the Pediatric Aerosol Research Laboratory, Arkansas Children's Hospital Research Institute, Little Rock, Arkansas.

Dr El Taoum presented a version of this paper at the ATS 2014 International Conference, held May 16-21, 2014, in San Diego, California.

This work was supported by the University of Arkansas for Medical Sciences College of Medicine Children's University Medical Group Fund Grant Program (036156). Dr Berlinski has disclosed relationships with Gilead, Philips, Genentech, Vertex, AbbVie, Aptalis, Janssen, Teva, and young children with different conditions. ${ }^{1-6}$ National and international guidelines recommend the use of inhaled albuterol, ipratropium bromide, and corticosteroids for asthma treatment. ${ }^{7,8}$ The guidelines also recommend the use of specific interfaces for different age groups and situations. Hypertonic saline has been used in several clinical trials in subjects with bronchiolitis, and inhaled antibiotics, inhaled $7 \%$ hypertonic saline, and dornase alfa are

\footnotetext{
Therapeutics Development Network. The other authors have disclosed no conflicts of interest.

Correspondence: Ariel Berlinski MD, Department of Pediatrics, Pulmonology Section, University of Arkansas for Medical Sciences, 1 Children's Way, Slot 512-17, Little Rock, AR 72202. E-mail: berlinskiariel@ uams.edu.
}

DOI: $10.4187 /$ respcare. 03606 
being used in infants and young children with cystic fibrosis. ${ }^{2-6}$

Several factors are known to affect drug delivery to a patient and can be divided into aerosol-related and patientrelated factors. ${ }^{9}$ Aerosol-related factors include type of aerosol generator, patient interface, particle size, treatment time, mask dead volume, type of power gas, and type of solution. ${ }^{10-14}$ Patient-related factors include differences in anatomy, breathing patterns, and behavioral challenges. ${ }^{15-18}$ Higher tidal volumes $\left(\mathrm{V}_{\mathrm{T}}\right)$ are associated with higher intrapulmonary deposition. ${ }^{19}$ On the other hand, behavioral challenges are crucial in infants and young children. Crying and lack of proper mask seal are known to decrease intrapulmonary deposition. ${ }^{17,18,20} \mathrm{An}$ in vivo study by Esposito-Festen et $\mathrm{al}^{21}$ demonstrated lack of acceptability and efficiency of aerosol delivery during sleep in a group of toddlers. This study highlighted the importance of interface acceptance to achieve good drug delivery.

There are different types of nebulizers available, including jet, ultrasonic, and vibrating mesh nebulizers. ${ }^{22}$ The jet nebulizer is the most commonly prescribed nebulizer. It utilizes a gas source to convert a liquid solution/suspension of a drug into an aerosol and propels it through the interface opening as a stream delivered to the patient's face. The ultrasonic nebulizer uses high frequency acoustical energy to convert a liquid into a mist. The vibrating mesh nebulizer generates a mist by squeezing the solution through a mesh with laser-drilled holes. This system uses electricity, not a gas source, to function. .,22,23 $^{2}$

Several authors have studied transnasal drug delivery with nasal cannulas using non-anatomically correct models. ${ }^{24,25}$ Other authors evaluated drug delivery using mouthbreathing models. ${ }^{11,26-28}$ Because infants and young children are obligate nose breathers, studies utilizing the nasal route for aerosol delivery are of importance. ${ }^{29-31}$ Recently, Amirav et $\mathrm{al}^{31}$ reported that nasal breathing resulted in larger lung deposition than mouth breathing in an in vivo study using airway replicas of 5-, 14-, and 20-month-old infants and toddlers. The authors showed a different response depending on the size of the airway models, with significantly higher aerosol delivery to the lower respiratory tract via the nasal route in comparison with the oral route in small and medium airway replicas. The same authors reported equivalent intrapulmonary deposition of a radiolabeled aerosol between a traditional mask and a newly designed mask that incorporates the use of a pacifier and provides nasal delivery. ${ }^{32}$

Although several patient interfaces aiming to provide transnasal drug delivery are available, little data comparing their efficiency are available. In this study, we compared the effects of varying interfaces, breathing patterns, and delivery devices on albuterol delivery via the transnasal route in anatomically correct models of a spontaneously breathing infant and child. We hypothesized that the

\section{QUICK LOOK}

\section{Current knowledge}

Aerosol therapy is a common drug delivery technique used in infants and children with respiratory disease. Aerosolized medications are commonly delivered via a mask or mouthpiece, which can be problematic in this group of obligate nose breathers. Aerosol therapy by the nasal route has not been well studied in this population, and multiple factors impact deposition and effectiveness.

\section{What this paper contributes to our knowledge}

In a pediatric model of the upper and lower airways, transnasal aerosol delivery to the lung was low, ranging from $0 \%$ to $3 \%$. A tight-fitting face mask was the most efficient interface with the infant and child breathing patterns, whereas no differences among interfaces were found with the newborn breathing pattern. The jet nebulizer was more efficient than the vibrating mesh nebulizer. The findings demonstrate the need to use anatomically correct airway models to study aerosol delivery in infants and children.

use of a vibrating mesh nebulizer, a tight-fitting face mask, and a head model and breathing pattern of an older child would result in higher lung doses. We also hypothesized that the use of an anatomically correct model would have a closer in vitro/in vivo correlation than the use of nonanatomically correct models.

\section{Methods}

The study was performed at the Pediatric Aerosol Research Laboratory of Arkansas Children's Hospital Research Institute in Little Rock, Arkansas.

\section{Delivery Devices}

Two different types of nebulizers were evaluated: a continuous output jet nebulizer (Hudson RCI Up-Draft II OptiNeb nebulizer, Teleflex Medical, Research Triangle Park, North Carolina) operated at $7 \mathrm{~L} / \mathrm{min}$ with wall air and a vibrating mesh nebulizer (Aeroneb Go, Aerogen, Galway, Ireland). Both nebulizers were loaded with albuterol sulfate nebulizer solution $(5 \mathrm{mg} / 3.5 \mathrm{~mL}$; Nephron Pharmaceuticals, Orlando, Florida) and operated for $5 \mathrm{~min}$. We used this dose to enhance our ability to recover drug from the filters. 


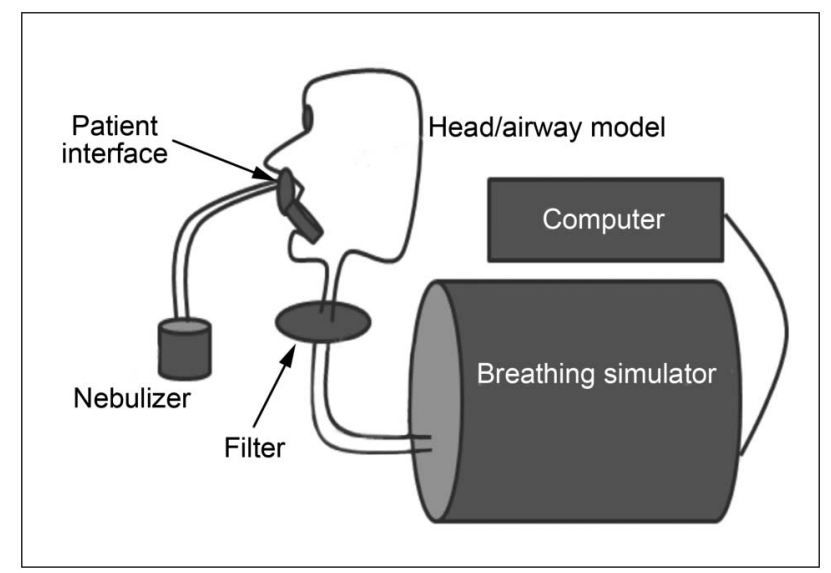

Fig. 1. Experimental setup to measure lung dose during simulated breathing.

Table 1. Breathing Patterns

\begin{tabular}{lcccc}
\hline \hline Breathing Pattern & $\begin{array}{c}\mathrm{V}_{\mathrm{T}} \\
(\mathrm{mL})\end{array}$ & $\begin{array}{c}\mathrm{f} \\
\text { (breaths/min) }\end{array}$ & $\begin{array}{c}\mathrm{T}_{\mathrm{I}} \\
(\mathrm{s})\end{array}$ & $\begin{array}{c}\mathrm{I}: \mathrm{E} \\
\text { Ratio }\end{array}$ \\
\hline Newborn & 25 & 40 & 0.4 & $1: 3$ \\
Infant & 50 & 30 & 0.5 & $1: 3$ \\
Child & 155 & 25 & 0.8 & $1: 2$ \\
& & & & \\
\hline $\mathrm{V}_{\mathrm{T}}=$ tidal volume & & & & \\
$\mathrm{f}=$ breathing frequency & & & & \\
$\mathrm{T}_{\mathrm{I}}=$ inspiratory time \\
$\mathrm{I}: \mathrm{E}=$ inspiratory-expiratory ratio
\end{tabular}

\section{Breathing Model}

The breathing model was composed of a breathing simulator connected in series to an anatomically correct head/airway model. A low dead-space filter holder was interposed between them (Fig. 1). The filter captured the lung dose that was expressed as a percentage of the loading dose. The 7-month-old head model was tested with all 3 breathing patterns, and the 5-y-old head model was tested only with the infant and child breathing patterns.

A computer-controlled syringe (Pari Compass, Munich, Germany) was used. This device allows programming of specific $\mathrm{V}_{\mathrm{T}}$, breathing frequency, and inspiratory-expiratory ratio. Delivered volumes were calibrated and verified at the beginning of each experiment using a mass flow meter (TSI 4043, TSI, Shoreview, Minnesota) and its associated software. ${ }^{14}$

Three different breathing patterns corresponding to a newborn, infant, and child were tested. The use of these breathing patterns has been recommended for evaluation of nebulized products intended for pediatric use (Table 1). ${ }^{33}$ All 3 breathing patterns were used with the 7-monthold head model, but only the infant and child breathing patterns were used with the 5-y-old head model. Magnetic resonance imaging and computed tomography scans of the head and neck of a 7-month-old infant and 5-y-old child were converted to tridimensional images, which were then printed as a 3 -dimensional image. ${ }^{15}$

\section{Interfaces}

The following interfaces were used: VixOne PediNeb pacifier with an angled connector or with a T-piece connector (DeVilbiss Healthcare, Somerset, Pennsylvania), pacifier adapter (B\&B Medical Technologies, Carlsbad, California), T-piece with a capped corrugated tube provided with the Hudson RCI Up-Draft II OptiNeb nebulizer, SootherMask (InspiRx, Somerset, New Jersey), and fish mask (Bubbles the Fish II, Pari Respiratory Equipment, Midlothian, Virginia) (Fig. 2). The latter was tested tightly fitted to the face model and placed $2 \mathrm{~cm}$ from the face. The fish mask was chosen because it is a front-loaded mask that allows a horizontal delivery of the mist directly to the face. This interface was proven to be more efficient than masks with other designs. ${ }^{11,12}$ Because the head models were constructed of a rigid material, the mask seal was achieved with putty. It was much harder to achieve a perfect seal with the SootherMask in comparison with the fish mask, most probably because of the very thin material used to make it.

The VixOne PediNeb pacifier with an angled connector or with a T-piece connector is composed of 2 parts. The first part is similar to and is shaped like a pacifier with a built-in funnel on top that allows delivery of the aerosol through the nostrils when the pacifier is in the mouth. The second part is unique to each interface; the angled connector is a short angled piece that connects the pacifier to the nebulizer, and the T-piece connector is a long vertical corrugated tube that connects the nebulizer to a T-piece and then the pacifier. The $\mathrm{B} \& \mathrm{~B}$ pacifier adapter is a corrugated tube connected to a funnel with 2 small adhesive wings that allow it to adhere to the patient's own pacifier and permits nasal delivery of the aerosol while the patient is sucking. The distance between the funnel on top of the pacifier is greater for the $\mathrm{B} \& \mathrm{~B}$ pacifier adapter than the VixOne PediNeb pacifier. The SootherMask is a proprietary mask with very low dead space that allows nasal aerosol delivery and is kept in place by the infant sucking his own pacifier, which can thread into it.

\section{Study Procedure}

The breathing model was assembled. A nebulizer was weighted, on a precision scale, dry $\left(\mathrm{W}_{\mathrm{dry}}\right)$ and after loading the albuterol sulfate $(5 \mathrm{mg} / 3.5 \mathrm{~mL})$ in the nebulizer $\left(\mathrm{W}_{\text {loaded }}\right)$. A nebulizer was connected to an interface. The position of the interface was adjusted to allow good alignment between the aerosol stream and the nostrils of the face model. The nebulizer was operated for 5 min and was then reweighted 


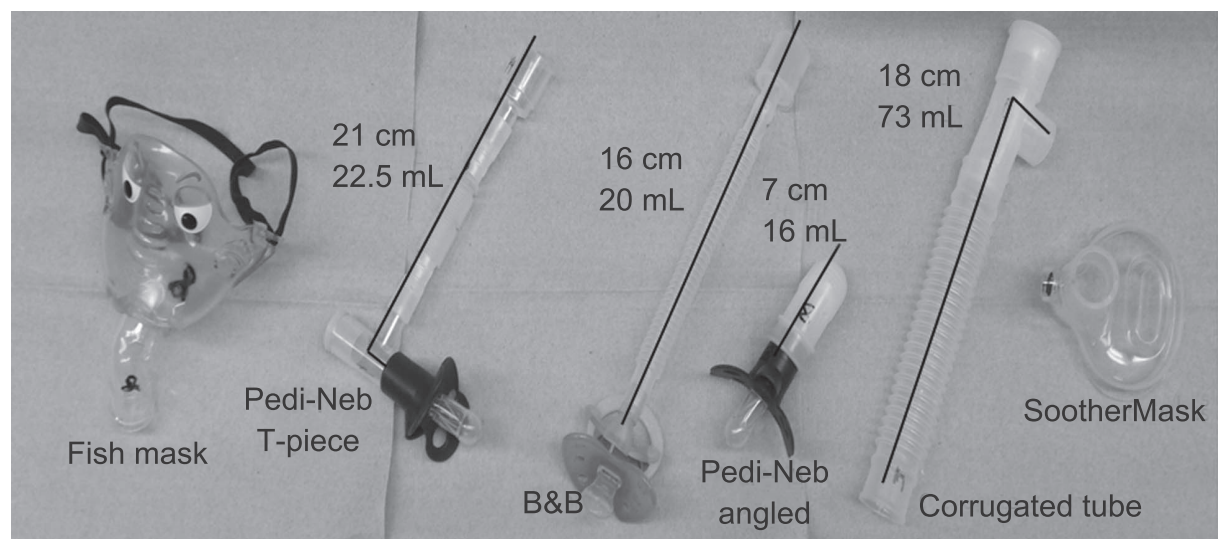

Fig. 2. Interfaces used in the study. The VixOne PediNeb pacifier is shown with both a T-piece connector and angled connector. B\&B = B\&B pacifier adapter; Corrugated Tube $=\mathrm{T}$-piece with a capped corrugated tube .

$\left(\mathrm{W}_{\text {final }}\right)$. Upon completion of the nebulization, $5 \mathrm{~mL}$ of ultrapure water were added to the nebulizer cup, the content was weighted $\left(\mathrm{W}_{\text {final }+5}\right)$, and the contents were swirled and tested for albuterol concentration. For the vibrating mesh nebulizer, $5 \mathrm{~mL}$ of ultrapure water were added to the base, and the content was swirled and tested for albuterol concentration. The filter was placed in a $50-\mathrm{mL}$ tube, and $10 \mathrm{~mL}$ of ultrapure water that had been previously used to wash the internal parts of the filter holder were added. The tube was vigorously shaken and vortexed before analyzing the washings. The amount of albuterol present in the filter and filter holder was expressed as a percentage of the nominal dose placed in the nebulizer $(5,000 \mu \mathrm{g})$ and termed lung dose. The amount of albuterol remaining in the nebulizer cup was calculated as follows: $\left(\mathrm{W}_{\text {final }+5}-\mathrm{W}_{\text {dry }}\right) \times$ albuterol concentration. Solution output was calculated by a gravimetric technique: $\mathrm{W}_{\text {loaded }}-\mathrm{W}_{\text {final }}$. The amounts of albuterol remaining in the cup and solution output were used as quality control indicators of nebulizer output. Because no differences were noted, any difference in lung dose could not be attributed to nebulizer performance, and therefore, the results are not reported. The study plan describes the different combinations of head model, interfaces, breathing patterns, and nebulizers (Fig. 3). All scenarios were tested one time using 4 different devices $(n=4)$. If lung dose was detected, a repeat series was done $(n=8)$.

\section{Albuterol Measurement}

Albuterol concentration was measured by spectrophotometry at $276 \mathrm{~nm}$ (BioMate $3 \mathrm{UV}$-visible spectrophotometer, Thermo Fisher Scientific, Waltham, Massachusetts). ${ }^{14}$

\section{Statistical Analysis}

Statistical analysis was performed with KaleidaGraph 4.1 (Synergy Software, Reading, Pennsylvania). To compare in-

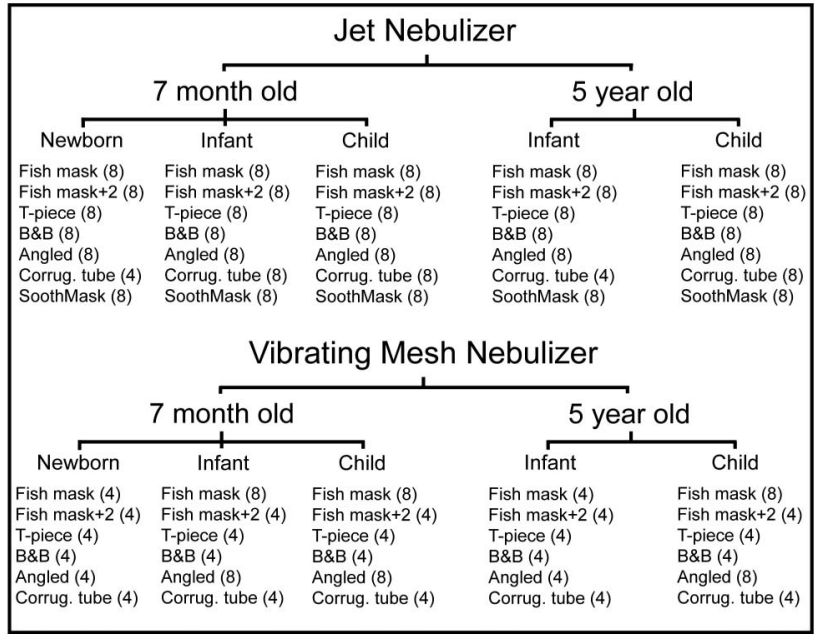

Fig. 3. Study plan. The $n$ for each scenario is in parentheses after each interface. Fish mask $+2=$ fish mask placed $2 \mathrm{~cm}$ from the face; T-piece $=$ VixOne PediNeb pacifier with a T-piece connector; $\mathrm{B} \& \mathrm{~B}=\mathrm{B} \& \mathrm{~B}$ pacifier adapter; Angled $=$ VixOne PediNeb pacifier with an angled connector; Corrug. Tube $=$ T-piece with a capped corrugated tube.

terfaces for each head model/breathing pattern/nebulizer combination, we used analysis of variance followed by the Dunnett test using a face mask as a control. To compare delivery devices for each head model/breathing pattern/interface combination and to compare head models for each nebulizer/ breathing pattern/interface combination, we used the $t$ test. To compare lung dose at different $\mathrm{V}_{\mathrm{T}}$ values for each head model/nebulizer/interface combination, we used analysis of variance followed by the Tukey test. Continuous variables were described using mean \pm SD. $P<.05$ was considered statistically significant.

\section{Results}

Data are summarized in Table 2 and Figures 4 and 5 . 
Table 2. Lung Dose

\begin{tabular}{|c|c|c|c|c|c|c|c|}
\hline \multirow{2}{*}{$\begin{array}{l}\text { Model/nebulizer/breathing } \\
\text { pattern }\end{array}$} & \multicolumn{7}{|c|}{ Lung Dose (\% of Nominal Dose) } \\
\hline & Fish Mask & Fish Mask +2 & T-Piece & $\mathrm{B} \& \mathrm{~B}$ & Angled & Corrugated Tube & SootherMask \\
\hline \multicolumn{8}{|l|}{ 7-mo-old head model } \\
\hline \multicolumn{8}{|l|}{ Jet } \\
\hline \multirow[t]{5}{*}{ Child } & $2.97 \pm 0.73$ & $2.06 \pm 0.36$ & $1 \pm 0.31$ & $0.97 \pm 0.55$ & $0.94 \pm 0.46$ & $1.97 \pm 0.26$ & $2.16 \pm 0.36$ \\
\hline & $.008^{*}$ & $<.001 *$ & $<.001 *$ & $<.001^{*}$ & $.62 *$ & $<.001 *$ & NA* \\
\hline & $.003 \dagger$ & $.001 \dagger$ & $.40 \dagger$ & $.39 \dagger$ & $.56 \dagger$ & $<.001 \dagger$ & $<.001 \dagger$ \\
\hline & $<.001 \ddagger$ & $<.001 \ddagger$ & $.003 \ddagger$ & $.43 \$$ & $.07 \ddagger$ & $<.001 \ddagger$ & $<.001 \ddagger$ \\
\hline & & $.001 \S$ & $<.001 \S$ & $<.001 \S$ & $<.001 \S$ & $.003 \S$ & $.005 \S$ \\
\hline \multirow[t]{4}{*}{ Infant } & $1.52 \pm 0.44$ & $0.73 \pm 0.3$ & $0.83 \pm 0.46$ & $1.22 \pm 0.57$ & $1.09 \pm 0.55$ & $0.49 \pm 0.2$ & $1.02 \pm 0.12$ \\
\hline & $.003 *$ & $<.001 *$ & $<.001 *$ & $<.001 *$ & $.003 *$ & $<.001 *$ & NA* \\
\hline & .002 & .61 & .15 & .08 & .031 & .007 & $<.001$ \\
\hline & & $.002 \S$ & $.008 \S$ & $.49 \S$ & $.17 \S$ & $<.001 \S$ & $.08 \S$ \\
\hline \multirow[t]{2}{*}{ Newborn } & $0.6 \pm 0.27$ & $0.65 \pm 0.32$ & $0.57 \pm 0.14$ & $0.78 \pm 0.36$ & $0.54 \pm 0.34$ & 0 & $0.07 \pm 0.20$ \\
\hline & $<.001 *$ & $<.001 *$ & $<.001 *$ & $<.001^{*}$ & $<.001^{*}$ & $* .99$ & *NA \\
\hline Vibrating mesh & & $.99 \S$ & $.99 \S$ & $.59 \S$ & $.99 \S$ & $.002 \S$ & $.001 \S$ \\
\hline \multirow[t]{4}{*}{ Child } & $1.9 \pm 00.66$ & 0 & 0 & 0 & $0.84 \pm 0.31$ & 0 & NA \\
\hline & $.008 \dagger$ & $.99 \dagger$ & $.99 \dagger$ & $.99 \dagger$ & $<.001 \dagger$ & $.99 \dagger$ & NA \\
\hline & $.002 \neq$ & $.99 \ddagger$ & $.99 \ddagger$ & $.99 \neq$ & .004 & $.99 \ddagger$ & NA \\
\hline & & $<.001 \S$ & $<.001 \S$ & $<.001 \S$ & $<.001 \S$ & $<.001 \S$ & NA \\
\hline \multirow[t]{3}{*}{ Infant } & $0.71 \pm 0.44$ & 0 & 0 & 0 & $0.13 \pm 0.15$ & 0 & NA \\
\hline & .01 & .99 & .99 & .99 & .12 & .99 & NA \\
\hline & & $<.001 \S$ & $<.001 \S$ & $<.001 \S$ & $<.001 \S$ & $<.001 \S$ & NA \\
\hline \multirow[t]{2}{*}{ Newborn } & 0 & 0 & 0 & 0 & 0 & 0 & NA \\
\hline & $.99 \S$ & $.99 \S$ & $.99 \S$ & $.99 \S$ & $.99 \S$ & $.99 \S$ & NA \\
\hline \multirow{2}{*}{\multicolumn{8}{|c|}{$\begin{array}{l}\text { 5-y-old head model } \\
\text { Jet }\end{array}$}} \\
\hline & & & & & & & \\
\hline \multirow[t]{4}{*}{ Child } & $2.96 \pm 0.62$ & $1.91 \pm 0.18$ & $0.97 \pm 0.46$ & $1.23 \pm 0.36$ & $1.22 \pm 0.57$ & $1.34 \pm 0.28$ & NA \\
\hline & $<.001 *$ & $<.001 *$ & $<.001^{*}$ & $<.001 *$ & $.006^{*}$ & $<.001 *$ & NA \\
\hline & $<.001 \dagger$ & $<.001 \dagger$ & $.04 \dagger$ & $.003 \dagger$ & $.003 \dagger$ & $<.001 \dagger$ & NA \\
\hline & & $.001 \S$ & $<.001 \S$ & $<.001 \S$ & $<.001 \S$ & $<.001 \S$ & NA \\
\hline \multirow[t]{3}{*}{ Infant } & $0.61 \pm 0.32$ & $0.14 \pm 0.33$ & $0.44 \pm 0.49$ & $0.53 \pm 0.41$ & $0.36 \pm 0.34$ & 0 & NA \\
\hline & $<.001 *$ & $<.001 *$ & $<.001 *$ & $<.001^{*}$ & $<.001 *$ & $.99 *$ & NA \\
\hline & & $.04 \S$ & $.79 \S$ & $.99 \S$ & $.48 \S$ & $.005 \S$ & NA \\
\hline \multicolumn{8}{|l|}{ Vibrating mesh } \\
\hline \multirow[t]{3}{*}{ Child } & $0.79 \pm 0.62$ & 0 & 0 & 0 & $0.42 \pm 0.39$ & 0 & NA \\
\hline & $.03 \dagger$ & $.99 \dagger$ & $.99 \dagger$ & $.99 \dagger$ & $.06 \dagger$ & $.99 \dagger$ & NA \\
\hline & & $<.001 \S$ & $<.001 \S$ & $<.001 \S$ & $.18 \S$ & $<.001 \S$ & NA \\
\hline \multirow[t]{2}{*}{ Infant } & 0 & 0 & 0 & 0 & 0 & 0 & NA \\
\hline & & $.99 \S$ & $.99 \S$ & $.99 \S$ & $.99 \S$ & $.99 \S$ & NA \\
\hline \multicolumn{8}{|c|}{ Values are mean $\pm \mathrm{SD}$. Boldface $P$ values are statistically significant. Significance level was set at $<.05$} \\
\hline \multicolumn{8}{|c|}{$\begin{array}{l}\text { * } P \text { values for the comparison of lung doses obtained with jet and vibrating mesh nebulizers for the specific breathing pattern/head model/interface }(t \text { test). } \\
\dagger P \text { values for the comparison of lung doses obtained with child and infant breathing patterns for the specific interface/nebulizer/head model (analysis of variance followed by Tukey test). } \\
¥ P \text { values for the comparison of lung doses obtained with child and newborn breathing patterns for the specific interface/nebulizer/head model (analysis of variance followed by the Tukey test). } \\
\S P \text { values for the comparison of lung doses obtained with different interfaces for the specific breathing pattern/head model/nebulizer (analysis of variance followed by the Dunnett test, using the fish } \\
\text { mask as a control). }\end{array}$} \\
\hline \multicolumn{8}{|c|}{$\begin{array}{l}P \text { values for the comparison of lung doses obtained with infant and newborn breathing patterns for the specific interface/nebulizer/head model (analysis of variance followed by the Tukey test). } \\
\text { Fish Mask }+2=\text { fish mask placed } 2 \mathrm{~cm} \text { from the face }\end{array}$} \\
\hline \multicolumn{8}{|c|}{$\begin{array}{l}\text { T-Piece }=\text { VixOne PediNeb pacifier with a T-piece connector } \\
\text { B\&B }=\text { B\&B pacifier adapter }\end{array}$} \\
\hline \multirow{2}{*}{\multicolumn{8}{|c|}{ B\&B = B\&B pacifier adapter }} \\
\hline & & & & & & & \\
\hline $\begin{array}{l}\text { Corrugated Tube }=\text { T-piece with a c } \\
\text { NA }=\text { not applicable }\end{array}$ & ped corrugated tube & & & & & & \\
\hline & & & & & & & \\
\hline
\end{tabular}



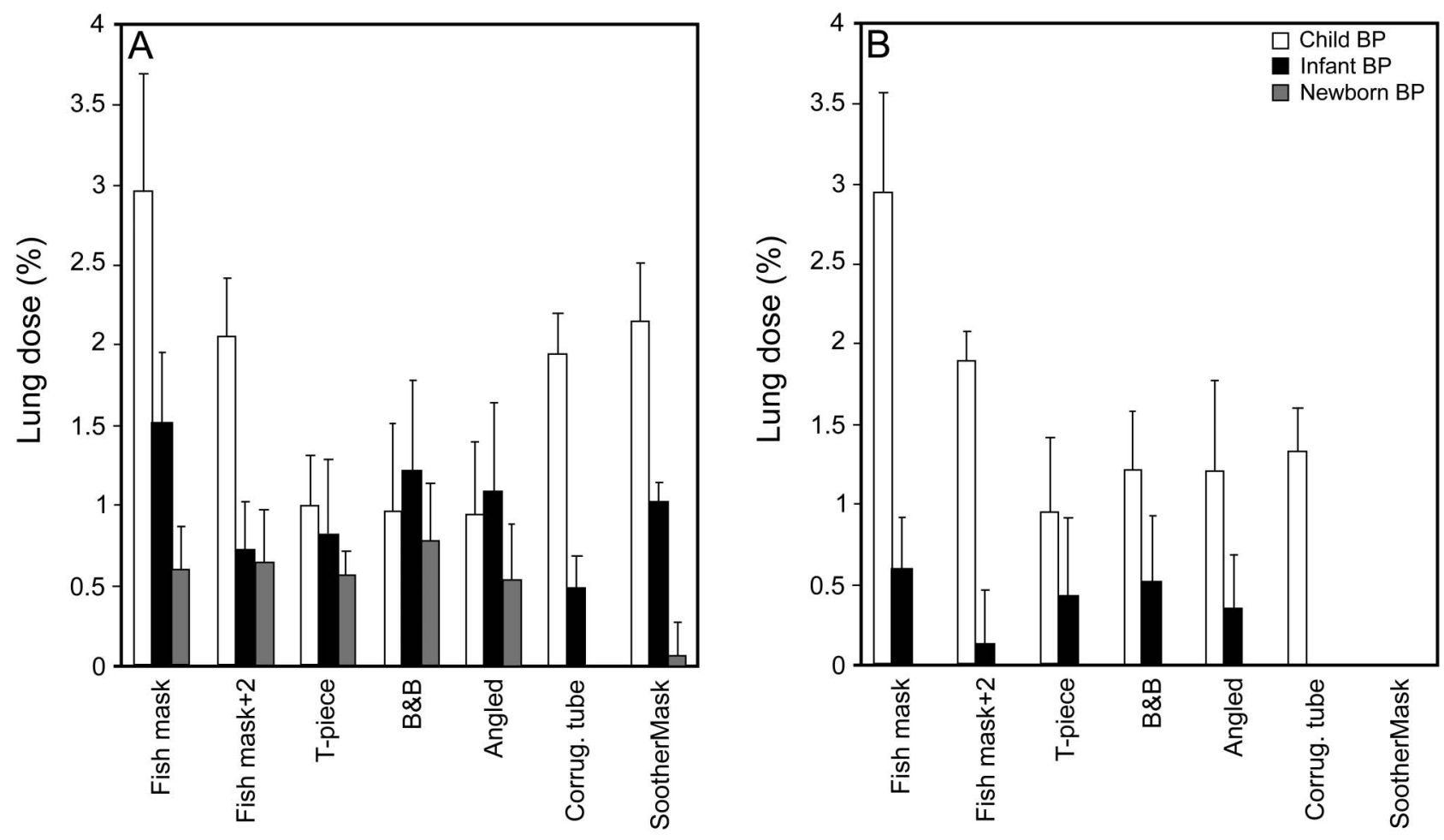

Fig. 4. Lung dose obtained via a jet nebulizer with infant $(A)$ and child $(B)$ model breathing patterns $(B P)$. Fish mask $+2=$ fish mask placed $2 \mathrm{~cm}$ from the face; T-piece $=$ VixOne PediNeb pacifier with a T-piece connector; B\&B = B\&B pacifier adapter; Angled $=$ VixOne PediNeb pacifier with an angled connector; Corrug. tube $=T$-piece with a capped corrugated tube. Results are expressed as a percentage of the loading dose. Bars represent the mean value, and error bars represent SD.

\section{Effect of Change in Interfaces on Lung Dose}

With the 7-Month-Old Head Model. The use of a jet nebulizer with the newborn breathing pattern resulted in similar lung doses in most interfaces $(P \geq .59)$, except for the T-piece with a capped corrugated tube and the SootherMask, which were lower $(P \leq .01)$. The infant breathing pattern resulted in similar lung doses with the fish mask tightly fitted to the face, B\&B pacifier adapter, PediNeb with an angled connector, and SootherMask $(P \geq .07)$, but lung doses were significantly lower with the other interfaces $(P \leq .008)$. The child breathing pattern resulted in a higher lung dose for the fish mask tightly fitted to the face than for all other interfaces $(P \leq .02)$.

With the vibrating mesh nebulizer, no drug was captured with any of the interfaces for the newborn breathing pattern. Effective lung doses were obtained only for the child and infant breathing patterns, achieved with the fish mask tightly fitted to the face and PediNeb with an angled connector. Higher lung doses were obtained with the fish mask tightly fitted to the face compared with the PediNeb with an angled connector $(P<.001)$ with both the infant and child breathing patterns.
With the 5-Year-Old Head Model. Using the jet nebulizer with the infant breathing pattern, lung doses were similar between the fish mask tightly fitted to the face and all other interfaces $(P>.48)$, except for the fish mask placed $2 \mathrm{~cm}$ away from the face and T-piece with a capped corrugated tube $(P=.04$ and $P=.005$, respectively). With the child breathing pattern, lung dose was significantly higher for the fish mask tightly fitted to the face in comparison with all other interfaces $(P<.001)$.

Using the vibrating mesh nebulizer, no drug was captured with the infant breathing pattern with any interface. With the child breathing pattern, effective lung doses were obtained only with the fish mask tightly fitted to the face and the PediNeb with an angled connector, with a 50\% decrease when switching from the mask to the connector. However, the difference did not achieve statistical significance due to the large variance in performance $(P=.18)$.

\section{Effect of Change in Delivery Devices on Lung Dose}

With the 7-Month-Old Head Model. With the newborn breathing pattern, an effective lung dose was obtained only with the jet nebulizer using all interfaces, except for the T-piece with a capped corrugated tube, which did not re- 

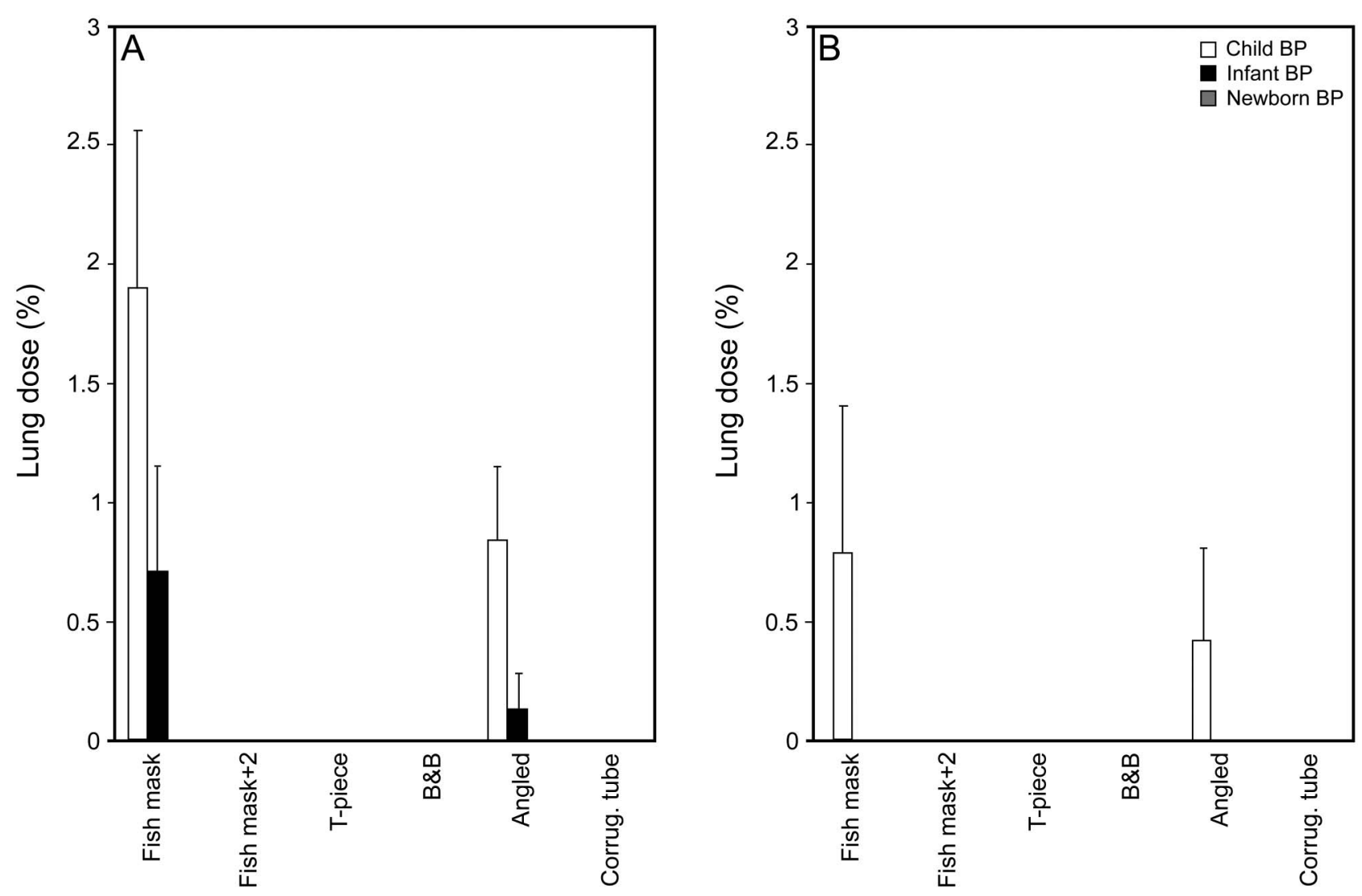

Fig. 5. Lung dose obtained via a vibrating mesh nebulizer in infant $(A)$ and child $(B)$ model breathing patterns $(B P)$. Fish mask $+2=$ fish mask placed $2 \mathrm{~cm}$ from the face; T-piece $=$ VixOne PediNeb pacifier with a T-piece connector; B\&B $=\mathrm{B} \& \mathrm{~B}$ pacifier adapter; Angled $=$ VixOne PediNeb pacifier with an angled connector; Corrug. tube $=$ T-piece with a capped corrugated tube. Results are expressed as a percentage of the loading dose. Bars represent the mean value, and error bars represent SD.

sult in any lung dose. No drug was captured with the vibrating mesh nebulizer.

With the infant breathing pattern, an effective lung dose was obtained with all interfaces for the jet nebulizer, but only with the fish mask tightly fitted to the face and the PediNeb with an angled connector for the vibrating mesh nebulizer. Using the fish mask, lung dose was decreased by $50 \%$ with the vibrating mesh nebulizer compared with the jet nebulizer $(P=.002)$ and by $93 \%$ when the PediNeb with an angled connector was used $(P=.003)$. With the child breathing pattern, an effective lung dose was obtained with all interfaces for the jet nebulizer, but only with the fish mask tightly fitted to the face and the PediNeb with an angled connector for the vibrating mesh nebulizer. Using the fish mask, lung dose was decreased by $63 \%$ with the vibrating mesh nebulizer compared with the jet nebulizer $(P=.008)$, but was similar for both nebulizers when the PediNeb with an angled connector was used $(P=.62)$.

With the 5-Year-Old Head Model. With the infant breathing pattern, an effective lung dose was obtained with all interfaces, except for the corrugated tube for the jet nebulizer, but no drug was captured for the vibrating mesh nebulizer. With the child breathing pattern, an effective lung dose was obtained with all interfaces for the jet nebulizer, but only with the face mask tightly fitted to the face and the PediNeb with an angled connector for the vibrating mesh nebulizer. Lung dose decreased by $73 \%$ and $66 \%$ with the vibrating mesh nebulizer compared with the jet nebulizer for the tightly fitted fish mask $(P<.001)$ and the PediNeb with an angled connector $(P=.006)$, respectively.

\section{Effect of Change in Airway Model on Lung Dose}

Using the Jet Nebulizer. With the child breathing pattern, lung dose was similar between airway models for all interfaces. With the infant breathing pattern, lung dose was reduced in the larger head model independently of the interface used, except for the corrugated tube with a capped T-piece $(P=.12)$.

Using the Vibrating Mesh Nebulizer. With the child breathing pattern, an effective lung dose was only ob- 
tained with the fish mask tightly fitted to the face and the PediNeb with an angled connector. Using the 5-yold head model resulted in 58\% and 50\% reduction in lung dose, respectively, compared with the 7-month-old head model. With the infant breathing pattern, an effective lung volume was obtained only using the fish mask tightly fitted to the face and the PediNeb with an angled connector for the 7-month-old head model. No drug was captured with any interface for the 5-y-old head model.

\section{Effect of Change in $V_{T}$ on Lung Dose}

With the 7-Month-Old Head Model. Using the jet nebulizer, lung dose was highest for the child breathing pattern and lowest for the newborn breathing pattern with the fish mask tightly fitted to the face and placed $2 \mathrm{~cm}$ from the face, the PediNeb with a T-piece with a capped corrugated tube, and the SootherMask. Lung dose was similar between the child and infant breathing patterns using the PediNeb with a T-piece, B\&B pacifier adapter, and PediNeb with an angled connector. Lung dose was similar between the infant and newborn breathing patterns for the face mask placed $2 \mathrm{~cm}$ from the face, PediNeb with a T-piece connector, and $\mathrm{B} \& \mathrm{~B}$ pacifier adapter.

Using the vibrating mesh nebulizer, an effective lung dose was obtained only with the infant and child breathing patterns using the fish mask tightly fitted to the face and the PediNeb with an angled connector. There was a 2.7fold and 6.5-fold difference between breathing patterns for the mask $(P=.002)$ and connector $(P=.004)$, respectively.

With the 5-Year-Old Head Model. A higher $\mathrm{V}_{\mathrm{T}}$ resulted in a higher lung dose for the jet and vibrating mesh nebulizers. The difference in lung dose between the child and infant breathing patterns reached statistical significance for the fish mask tightly fitted to the face $(P=.03)$, but not for the PediNeb with an angled connector $(P=.06)$ due to its large SD. Of note, no drug was captured with the infant breathing pattern and the vibrating mesh nebulizer.

\section{Discussion}

In this in vitro study, we compared the effect of varying interfaces, delivery devices, breathing patterns, and age on lung dose of albuterol delivered via the transnasal route in anatomically correct models of a spontaneously breathing infant and child. We found that a jet nebulizer was more efficient than a vibrating mesh nebulizer and that a tightfitting face mask was the most efficient interface. We also found that, in general, a higher $\mathrm{V}_{\mathrm{T}}$ is associated with a higher lung dose and that transnasal delivery is more efficient in infants than in older children.

\section{Interfaces}

Our data confirmed that a tight-fitting, front-loaded mask (Bubbles the Fish II) resulted in a significantly higher lung dose than other interfaces. ${ }^{11,12,27}$ Lin et al ${ }^{11}$ compared the effect of front-loaded versus angle-loaded and bottomloaded masks on inhaled mass behind the oral opening of a mannequin face when delivering the aerosol with a jet nebulizer placed 0,1 , and $2 \mathrm{~cm}$ from the face using an infant breathing pattern $\left(\mathrm{V}_{\mathrm{T}}\right.$ of $60 \mathrm{~mL}$, breathing frequency of $20 \mathrm{breaths} / \mathrm{min}$, inspiratory time of $0.7 \mathrm{~s}$ ). The frontloaded nebulizer was the most efficient interface and showed a $38 \%$ decrease in inhaled mass when the mask was moved $2 \mathrm{~cm}$ from the face. The authors reported an inhaled mass that was 2.6-fold higher than ours. We speculate that this was due to the difference in the experimental design; in our study, we used an anatomically correct model of the face and airways, and our lung dose corresponded to the drug captured at the level of the carina. In their study, they instead captured the drug behind the mouth, therefore avoiding the upper airway filter. Using a similar experimental setup (drug captured behind the mouth and same breathing pattern), Mansour et $\mathrm{al}^{27}$ reported a 3.8-fold higher inhaled mass than ours and a 51\% decrease in inhaled mass when the mask was moved $2 \mathrm{~cm}$ from the face. As with Lin et al, ${ }^{11}$ we speculate that these differences were due to a different experimental setup that includes the use of an oral model, which overestimates drug delivery to children.

The SootherMask was less efficient than the fish mask. ${ }^{31}$ We speculate that this could have been due in part to the softness of the material and the difficulty in obtaining a good seal. Amirav et $\mathrm{al}^{31}$ also studied lung dose in anatomically correct infant and toddler models. Their model had a soft face, breathing patterns with a prolonged inspiratory time, and also a soft mist inhaler. These differences preclude us from comparing results between studies.

Chua et $\mathrm{al}^{16}$ reported lung deposition of a radiolabeled aerosol in infants and children with cystic fibrosis. They found that lung deposition was $1.3 \%$ in infants (mean age of $0.8 \mathrm{y}$ ) and $2.7 \%$ in older children (mean age of $10.8 \mathrm{y}$ ) when nasal breathing was used. Amirav et al ${ }^{31}$ compared intrapulmonary deposition of a radiolabeled aerosol in 12 infants (6 mo old) using a traditional face mask and the SootherMask. They reported equivalent lung deposition between both interfaces $(1.65 \%)$. The results from both studies are in agreement with our in vitro results, with $1 \%$ lung dose for the SootherMask and $1.4 \%$ for the fish mask with the infant breathing pattern and a 7-month-old head model and $2.96 \%$ for the fish mask using the child breathing pattern and 5-y-old head model. These findings underscore the importance of using anatomically correct models to study aerosol delivery in infants and children. As stated 
previously, other non-anatomically correct models overestimate drug delivery to infants and children. ${ }^{11,12,26-28}$

Three other nasal interfaces were used: the VixOne PediNeb pacifier with an angled connector or with a T-piece connector and the B\&B pacifier adapter. With the jet nebulizer, the nasal interfaces showed equivalent lung dose to the face mask placed $2 \mathrm{~cm}$ from the face when used with the infant and newborn breathing patterns, but lower lung dose with the child breathing pattern.

In our study, the use of a jet nebulizer with a T-piece and a capped corrugated tube placed $2 \mathrm{~cm}$ from the face resulted in an equivalent lung dose compared with the fish mask placed $2 \mathrm{~cm}$ from the face. In another study, a capped T-piece was found to be more efficient in delivering the aerosol to a filter placed at the inlet of a pediatric test lung $\left(\mathrm{V}_{\mathrm{T}}\right.$ of $60 \mathrm{~mL}$, breathing frequency of 20 breaths/min, inspiratory time of $1 \mathrm{~s}$ ) and placed at 0,1 , and $2 \mathrm{~cm}$ from the face. ${ }^{26}$ The authors reported an inhaled mass that was 7-fold higher than ours (7-mo-old head/infant breathing pattern). We speculate that the distance from the aerosol source to the nose increased by the corrugated tube (see Fig. 2), difference in inspiratory time, and use of an anatomically correct airway model were responsible for this difference.

With the vibrating mesh nebulizer, a detectable lung dose was obtained with the fish mask tightly fitted to the face and the PediNeb pacifier with an angled connector only and was less efficient than with the jet nebulizer. These findings are in contrast to those of Tiemersma et al, ${ }^{30}$ who studied aerosol delivery via jet and vibrating mesh nebulizers using an anatomically correct model of a premature infant. We speculate that the difference between both studies could be multifactorial. Our study used a longer interface and a larger head model. Findings from their study showed that the addition of an extension elbow caused significant lung dose reduction. Although we initially thought that equipment dead space could be responsible, we found that distance between the aerosol generator and the nostril was the determinant factor (see Fig. 2). This finding is in agreement with another study that showed that decreasing the dead space of the mask in nebulized therapy did not increase the amount of aerosol captured at the mouth opening. ${ }^{19}$

\section{Delivery Devices}

Our study showed that a higher lung dose was obtained with a jet nebulizer than with a vibrating mesh nebulizer with all interfaces and all breathing patterns. Skaria and Smaldone ${ }^{10}$ showed equivalent inhaled mass (20\% of nebulizer charge) for jet and vibrating mesh nebulizers in an in vitro model. They used a breathing pattern of a patient with COPD $\left(\mathrm{V}_{\mathrm{T}}\right.$ of $450 \mathrm{~mL}$, breathing frequency of 15 breaths/min).The inhaled mass in their study was much higher than our lung dose. We speculate that it is due to the difference in the experimental design. In our study, we used an anatomically correct model of the face and airways, and the lung dose corresponded to the drug captured at the level of the carina, whereas in their study, the drug was captured behind the mouth, and a higher $\mathrm{V}_{\mathrm{T}}$ was used. Tiemersma et $\mathrm{al}^{30}$ compared jet nebulizer and vibrating mesh nebulizers in a premature infant nose/throat model. Lung dose was $1.5 \%$ and $18-20.6 \%$ for jet nebulizer and vibrating mesh nebulizers, respectively. We speculate that our opposite findings on the effect of nebulizers on lung dose are due to experimental design, mainly the difference in distance between the nebulizer and face. Of note, in the same study, adding an elbow extension between the vibrating mesh nebulizer and model significantly decreased the lung dose. These findings highlight the importance of the choice of nebulizer/interface combination in drug delivery efficiency.

Our findings also disagree with another study that compared drug output during mechanical ventilation. ${ }^{34}$ The main difference that could explain these conflicting results is that drug delivery during mechanical ventilation using vibrating mesh devices is aided by the presence of flow during the exhalation phase (bias flow), and our model was not. Our findings also contradict those of Bhashyam et al, ${ }^{24}$ who studied aerosol delivery through a heated flow nasal cannula operated at $3 \mathrm{~L} / \mathrm{min}$ using a vibrating mesh nebulizer. They used a non-anatomically correct model and reported lung doses of $18.6 \%$ and $25.4 \%$ of the loading dose for infant and pediatric cannulas, respectively. The authors used only the inspiratory flow generated by the breathing simulator, but did not specify the breathing parameters. We speculate that the differences between both studies could be related to the fact that they used bias flow ( $3 \mathrm{~L} / \mathrm{min}$ ), did not release the expiration in the system, and used a non-anatomically correct model. Ari et $\mathrm{al}^{25} \mathrm{com}$ pared aerosol delivery through a heated flow nasal cannula using a vibrating mesh nebulizer with bias flows of 3 and $6 \mathrm{~L} / \mathrm{min}$. The authors used a pediatric breathing pattern $\left(\mathrm{V}_{\mathrm{T}}\right.$ of $100 \mathrm{~mL}$, breathing frequency of 20 breaths $/ \mathrm{min}$, inspiratory time of $1 \mathrm{~s}$ ), and a non-anatomically correct model allowed a straight path of the aerosol between the cannula and artificial nares. The authors reported $10.65 \%$ and $1.95 \%$ deposition, respectively, when bias flows of 3 and $6 \mathrm{~L} / \mathrm{min}$ were used. We speculate that the differences in experimental setup (bias flow and type of model) are responsible for the large differences noted in deposited dose.

\section{Head Model}

We found that the effect of head model on lung dose was dependent on the breathing pattern used. There was no significant difference in lung dose between both airway 
models for almost all interfaces when using the child breathing pattern combined with the jet nebulizer. However, lung dose was higher with the younger model compared with the older model with the infant breathing pattern. We speculate that the difference in dead space was responsible for this behavior. However, we performed a series of studies using a $\mathrm{V}_{\mathrm{T}}$ adjusted for the dead space of the models (22 and $10 \mathrm{~mL}$ for the 5-y-old and 7-mo-old models, respectively) that did not show any difference. This is in agreement with Amirav et al, ${ }^{31}$ who reported that differences in dead space of airway models did not explain differences in lung dose.

Our results show how the choice of head model and breathing patterns significantly affects lung dose results. This highlights the need for standardized representative breathing patterns and face/oronasal models to optimize the in vitro studies that closely resemble in vivo studies.

$\mathbf{V}_{\mathbf{T}}$

In our study, lung dose increased with increasing $\mathrm{V}_{\mathrm{T}}$. This is in agreement with Amirav et al, ${ }^{31}$ who compared lung dose obtained with a soft mist inhaler at different $V_{T}$ values with 3 different anatomically correct airway models using oral and nasal inhalation. Using the nasal route, the change from low to high $\mathrm{V}_{\mathrm{T}}$ increased lung dose by $89 \%$ and $120 \%$ with their 5-month-old and 14-month-old models, respectively, and by $112 \%$ with our 7 -month-old model. However, these results are not in agreement with those obtained by Laube et al, ${ }^{29}$ who reported no significant difference in lung dose at 3 different $\mathrm{V}_{\mathrm{T}}$ values $(50,100$, and $200 \mathrm{~mL}$ ) using a face mask and anatomically correct head model of a 9-month-old infant without an oral opening. They obtained 3-fold and 5-fold higher lung doses compared with our combinations of a 7-month-old head model, jet nebulizer, and child and infant breathing patterns, respectively. We speculate that the difference is due to variations in study design, including different head models and different nebulizers.

\section{Limitations}

This study had several limitations. In vitro models that use filters to capture the drug overestimate lung dose because they report a value that includes both inhaled lung dose and part of the exhaled lung dose. However, this is a widely accepted methodology. We used a rigid face model, which could have affected the mask seal despite the use of putty. This may have affected only the measurements obtained with a face mask. Also, the model is static and does not move like a child, resulting in overestimation of the lung dose since movement will cause misalignment of the aerosol stream and nostrils.

\section{Conclusions}

Lung dose by transnasal aerosol delivery was low, ranging from $0 \%$ to $3 \%$. Careful pairing of the aerosol generator and interface is very important during transnasal aerosol delivery. A tight-fitting face mask was the most efficient interface in the infant and child breathing patterns; however, no differences among interfaces were found with the newborn breathing pattern. The jet nebulizer was more efficient than the vibrating mesh nebulizer. The poor performance of the latter seems to be related to the distance between the nebulizer and face. In our model, breathing pattern affected lung dose. At low $\mathrm{V}_{\mathrm{T}}$, lung dose was similar regardless of the interface used. Head model and breathing patterns significantly affected lung dose results. Our findings underscore the need to use anatomically correct airway models to study aerosol delivery in infants and children. In vivo studies evaluating the acceptance by infant and children of the different interfaces are needed.

\section{REFERENCES}

1. Mogayzel PJ Jr, Naureckas ET, Robinson KA, Mueller G, Hadjiliadis D, Hoag JB, et al. Cystic fibrosis pulmonary guidelines. Chronic medications for maintenance of lung health. Am J Respir Crit Care Med 2013;187(7):680-689.

2. Treggiari MM, Retsch-Bogart G, Mayer-Hamblett N, Khan U, Kulich M, Kronmal R, et al. Comparative efficacy and safety of 4 randomized regimens to treat early Pseudomonas aeruginosa infection in children with cystic fibrosis. Arch Pediatr Adolesc Med 2011; 165(9):847-856.

3. Rosenfeld M, Ratjen F, Brumback L, Daniel S, Rowbotham R, McNamara $\mathrm{S}$, et al. Inhaled hypertonic saline in infants and children younger than 6 years with cystic fibrosis: the ISIS randomized controlled trial. JAMA 2012;307(21):2269-2277.

4. Wagener JS, Rock MJ, McCubbin MM, Hamilton SD, Johnson CA, Ahrens RC. Aerosol delivery and safety of recombinant human deoxyribonuclease in young children with cystic fibrosis: a bronchoscopic study. Pulmozyme Pediatric Broncoscopy Study Group. J Pediatr 1998;133(4):486-491.

5. American Academy of Pediatrics Subcommittee on Diagnosis and Management of Bronchiolitis. Diagnosis and management of bronchiolitis. Pediatrics 2006;118(4):1774-1793.

6. Chen YJ, Lee WL, Wang CM, Chou HH. Nebulized hypertonic saline treatment reduces both rate and duration of hospitalization for acute bronchiolitis in infants: an update meta-analysis. Pediatr Neonatol 2014;55(6):431-438

7. From the Global Strategy for Asthma Management and Prevention, Global Initiative for Asthma (GINA) 2014. http:// www.ginasthma.org/. Accessed June 15, 2014.

8. Expert Panel Report 3 (EPR3): guidelines for the diagnosis and management of asthma. Bethesda, MD: National Heart, Lung, and Blood Institute; 2007.

9. Laube BL, Janssens HM, de Jongh FH, Devadason SG, Dhand R, Diot $\mathrm{P}$, et al. What the pulmonary specialist should know about the new inhalation therapies. Eur Respir J 2011;37(6):1308-1331.

10. Skaria S, Smaldone G. Omron NE U22: comparison between vibrating mesh and jet nebulizer. J Aerosol Med Pulm Drug Deliv 2010; 23(3):173-180. 
11. Lin HL, Restrepo RD, Gardenhire DS, Rau JL. Effect of face mask design on inhaled mass of nebulized albuterol, using a pediatric breathing model. Respir Care 2007;52(8):1021-1026.

12. Harris KW, Smaldone GC. Facial and ocular deposition of nebulized budesonide: effect of face mask design. Chest 2008;133(2):482-488.

13. Janssens HM, van der Wiel EC, Verbraak AF, de Jongste JC, Merkus PJ, Tiddens HA. Aerosol therapy and the fighting toddler: is administration during sleep an alternative? J Aerosol Med 2003;16(4):395400.

14. Chavez A, McCracken A, Berlinski A. Effect of face mask static dead volume, respiratory rate and tidal volume on inhaled albuterol delivery. Pediatr Pulmonol 2010;45(3):224-229.

15. Xi J, Berlinski A, Zhou Y, Greenberg B, Ou X. Breathing resistance and ultrafine particle deposition in nasal-laryngeal airways of a newborn, an infant, a child, and an adult. Ann Biomed Eng 2012;40(12): 2579-2595.

16. Chua HL, Collis GG, Newbury AM, Chan K, Bower GD, Sly PD, Le Souef PN. The influence of age on aerosol deposition in children with cystic fibrosis. Eur Respir J 1994;7(12):2185-2191.

17. Iles R, Lister P, Edmunds AT. Crying significantly reduces absorption of aerosolised drug in infants. Arch Dis Child 1999;81(2):163165.

18. Murakami G, Igarashi T, Adachi Y, Matsuno M, Sawai M, Yoshizumi A, Okada T. Measurement of bronchial hyperreactivity in infants and preschool children using a new method. Ann Allergy 1990; 64(4):383-387.

19. Berlinski A. Effect of dead space and occlusion of mask holes on delivery of nebulized albuterol. Respir Care 2014;59(8):1228-1232.

20. Esposito-Festen JE, Ates B, van Vliet FJ, Verbraak AF, de Jongste JC, Tiddens HA. Effect of a facemask leak on aerosol delivery from a pMDI-spacer system. J Aerosol Med 2004;17(1):1-6.

21. Esposito-Festen J, Ijsselstijn H, Hop W, van Vliet F, de Jongste J, Tiddens H. Aerosol therapy by pressured metered-dose inhaler-spacer in sleeping young children: to do or not to do? Chest 2006;130(2): 487-492.

22. Dolovich MB, Dhand R. Aerosol drug delivery: developments in device design and clinical use. Lancet 2011;377(9770):1032-1045.

23. Hess DR. Aerosol delivery devices in the treatment of asthma. Respir Care 2008;53(6):699-723; discussion 723-725.
24. Bhashyam AR, Wolf MT, Marcinkowski AL, Saville A, Thomas K, Carcillo JA, Corcoran TE. Aerosol delivery through nasal cannulas: an in vitro study. J Aerosol Med Pulm Drug Deliv 2008;21(2):181188.

25. Ari A, Harwood R, Sheard M, Dailey P, Fink JB. In vitro comparison of heliox and oxygen in aerosol delivery using pediatric high flow nasal cannula. Pediatr Pulmonol 2011;46(8):795-801.

26. Restrepo RD, Dickson SK, Rau JL, Gardenhire DS. An investigation of nebulized bronchodilator delivery using a pediatric lung model of spontaneous breathing. Respir Care 2006;51(1):56-61.

27. Mansour MM, Smaldone GC. Blow-by as potential therapy for uncooperative children: an in-vitro study. Respir Care 2012;57(12): 2004-2011.

28. Sangwan S, Gurses BK, Smaldone GC. Facemasks and facial deposition of aerosols. Pediatr Pulmonol 2004;37(5):447-452.

29. Laube BL, Sharpless G, Shermer C, Nasir O, Sullivan V, Powell K. Deposition of albuterol generated by pneumatic nebulizer in the Sophia anatomical infant nose-throat (SAINT) model. Pharm Res 2010;27(8):1722-1729.

30. Tiemersma S, Minocchieri S, van Lingen RA, Nelle M, Devadason SG. Vibrating membrane devices deliver aerosols more efficiently than standard devices: a study in a neonatal upper airway model. J Aerosol Med Pulm Drug Deliv 2013;26(5):280-286.

31. Amirav I, Borojeni AA, Halamish A, Newhouse MT, Golshahi L. Nasal versus oral aerosol delivery to the "lungs" in infants and toddlers. Pediatr Pulmonol 2014 [Epub ahead of print] doi: 10.1002/ ppul.22999.

32. Amirav I, Luder A, Chleechel A, Newhouse MT, Gorenberg M. Lung aerosol deposition in suckling infants. Arch Dis Child 2012; 97(6):497-501.

33. Jauering J, Mitchell J, Berg E, Dennis J, Kreher C, Lamb P, et al. Position paper: recommendation on the adoption of breathing patterns for infants and small children in general. Chapter 2.9.44. Preparations for nebulization. Pharmeur Sci Notes 2008;(1):3132.

34. Berlinski A, Willis JR. Albuterol delivery by 4 different nebulizers placed in 4 different positions in a pediatric ventilator in vitro model. Respir Care 2013;58(7):1124-1133. 